\title{
Detecting and Characterizing the Fabella with High Frame-Rate Ultrasound Imaging
}

\author{
Michael A. Berthaume ${ }^{1}$, Matthieu Toulemonde ${ }^{2}$, Laura Peralta ${ }^{3}$, Kirsten Christensen-Jeffries ${ }^{3}$, Enrico Grisan ${ }^{4}$ \\ and Sevan Harput ${ }^{5}$ \\ ${ }^{1}$ Division of Mechanical Engineering and Design, London South Bank University, London, SE1 0AA, UK \\ ${ }^{2}$ ULIS Group, Department of Bioengineering, Imperial College London, London, SW7 2BP, UK \\ ${ }^{3}$ Biomedical Engineering Department, School of Biomedical Engineering \& Imaging Sciences, King's College London, SE1 7EH, UK \\ ${ }^{4}$ Division of Computer Science and Informatics, London South Bank University, London, SE1 0AA, UK \\ ${ }^{5}$ Division of Electrical and Electronic Engineering, London South Bank University, London, SE1 0AA, UK \\ E-mail: harputs@1sbu.ac.uk
}

\begin{abstract}
The fabella is a sesamoid bone usually located in the tendon of the lateral head of the gastrocnemius muscle, behind the knee joint. Prevalence rates in human populations vary widely with an average of $\mathbf{4 2 . 5 \%}$ people having a fabella. Clinically, it is associated with a number of knee ailments, most notably the osteoarthritis of the knee and generalized knee pain (i.e., fabella syndrome). As the function of the fabella remains unknown, the biomechanical consequences of fabella presence/absence can only be speculated. Successfully detecting the fabella, measuring its size and determining its shape, are off importance for clinical and evolutionary researchers. In this work, we compare plane wave imaging with conventional focused imaging and evaluate their performance for detecting and characterizing the fabella.
\end{abstract}

\section{INTRODUCTION}

The fabella is a sesamoid bone usually located inside the tendon of the calf muscle behind the knee joint; specifically inside the lateral head of the gastrocnemius muscle behind the lateral femoral condyle as illustrated in Fig. 1. In humans, fabellae are 3.5 times more common today than they were a century ago [1].The presence or absence of the fabella is due to a combination of genetic and environmental factors, and the increase over the last century may be linked to the global increase in nutrition, and consequently height and weight [2]. A recently published meta-analysis on fabella prevalence rate showed that on average $42.5 \%$ of people worldwide have a fabella [2].

The function of the fabella remains unknown, but it has been speculated that the fabella serves as a stabilizer of the knee [3], [4]. However, clinically the presence of fabella is associated with several conditions and diseases. The most common ones are the osteoarthritis and the fabella syndrome, which is knee pain caused by the presence fabella [5], [6]. Interestingly, knees with osteoarthritis are twice as likely to have a fabella than knees without osteoarthritis, although no causal link has been established [7]. However, it is still not proven that if fabella is causative of osteoarthritis or arising from osteoarthritis [1].

The presence of fabella is usually determined through imaging or dissection. Although dissection has a higher detection rate, the burden of the surgery usually makes it impractical

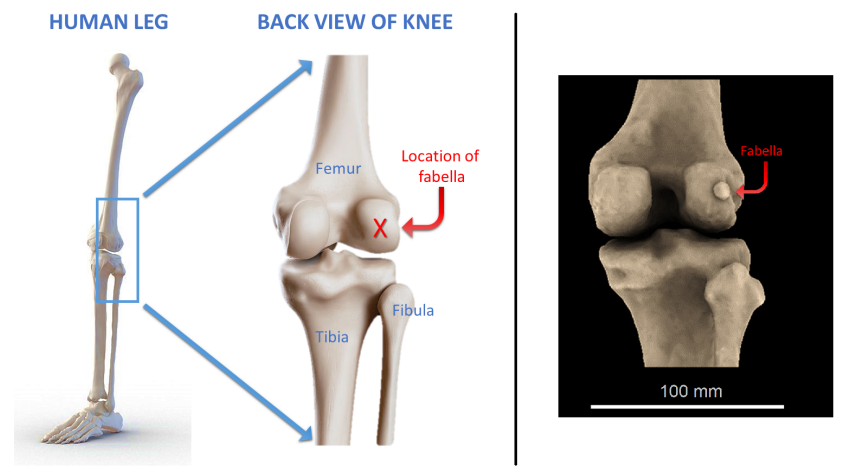

Fig. 1. (Left) Anatomical illustration showing the location of the fabella. (Right) CT scan of a human knee with fabella from [2].

to perform a large independent study on the prevalence rate of fabella. The commonly used imaging meth-ods to detect fabellae are X-ray, computed tomography (CT) or magnetic resonance imaging (MRI) scans, which can have low sensitivity, as fabellae can be cartilaginous/ have low density meaning their presence is not always be detected by X-ray or by a CT scans [8]. Scan resolution and knee position during scan can also make it difficult to detect small fabellae n MRI scans [9]. A study showed that over $57 \%$ of cases where fabellae were detectable by dissection were not detectable by radiograph due to low density or small size [10]. It is possible to employ ultrasound imaging to monitor fabellae, but the use of ultrasound for the detection and characterization of the fabella is only reported in a few studies [11]-[14].

Although there has been a lot of progress in bone imaging, most of the current research is focusing on measuring bone cortical thickness and estimating bone parameters, such as attenuation, bone mineral density and porosity [15]-[20]. This research differs from the aforementioned studies as it aims to accurately detect and calculate the dimensions of a small sesamoid bone: the fabella. To achieve this, this study first verifies the feasibility of using plane wave ultrasound imaging for characterization of fabella. This study also combines two 


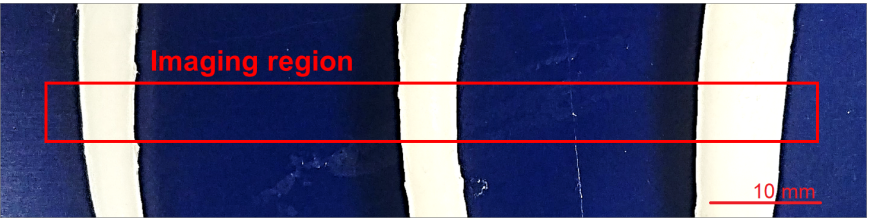

Fig. 2. Optical image of the ex vivo swine ribs (in white) placed on an acoustic absorber (in blue). Each ultrasound measurement was performed with 3 different swine ribs to reduce the total imaging time.

existing coherence factors to increase the beamforming performance and offers other potential data processing methods.

\section{Materials And Methods}

\section{A. Experimental Setup}

The imaging setup consists of a ULA-OP 256 system and a $4 \mathrm{MHz}$ 144-element linear array transducer (LA332, Esaote, Italy) [21], [22]. Ex vivo swine bones $(n=12)$ are used as model for the fabella given their similarities in bone architecture (thin cortical shell surrounding a trabecular interior) and similar dimensions (here, diameter $=4.2-8.5$ mm, Fig. 2). Two different imaging modalities were used: first, conventional linear scans with line-by-line scanning with an aperture size of 64 elements focused at $40 \mathrm{~mm}$. Second, compounded plane wave imaging (CPWI) was performed with an angle range of $24^{\circ}$, step size $0.5^{\circ}$, and at a pulse repetition frequency of $5 \mathrm{kHz}$.

\section{B. Data Processing}

Linear scan and CPWI images were beamformed using delay and sum beamforming. In addition to this, element coherence factor $(\mathrm{ECF})$ and angular coherence factor (ACF) methods were implemented to improve beamforming performance and reduce the image artefacts generated by highly reflective bone tissue. The ECF is based on the acoustic sub-aperture processing method, where the B-mode image is beamformed twice by using the odd and even elements separately and combined to extract the coherence [23]. The ACF is based on the accumulated angle factor, which is customized for bone surface enhancement as explained in [24].

A segmentation algorithm based on Gaussian Mixtures was used to detect the borders of the bone tissue and the segmented images were used to estimate bone dimensions [25]. Since the beamforming was performed using the speed of sound in water, the measured bone thickness were re-scaled according to the speed of sound in swine ribs. The sound speed in swine ribs was measured as $2217 \mathrm{~m} / \mathrm{s}$ using focused waves at the same cross section and orientation as the imaging setup. The estimated bone dimensions from ultrasound images were compared with the ground truth, which was measured by callipers (precision $\pm 0.01 \mathrm{~mm}$ ). The performance of each method was evaluated by their relative error in their estimates.

\section{RESULTS}

Among all CPWI images (Fig. 3 A-D) the ACF and ECF combination had the best overall performance. The delay and
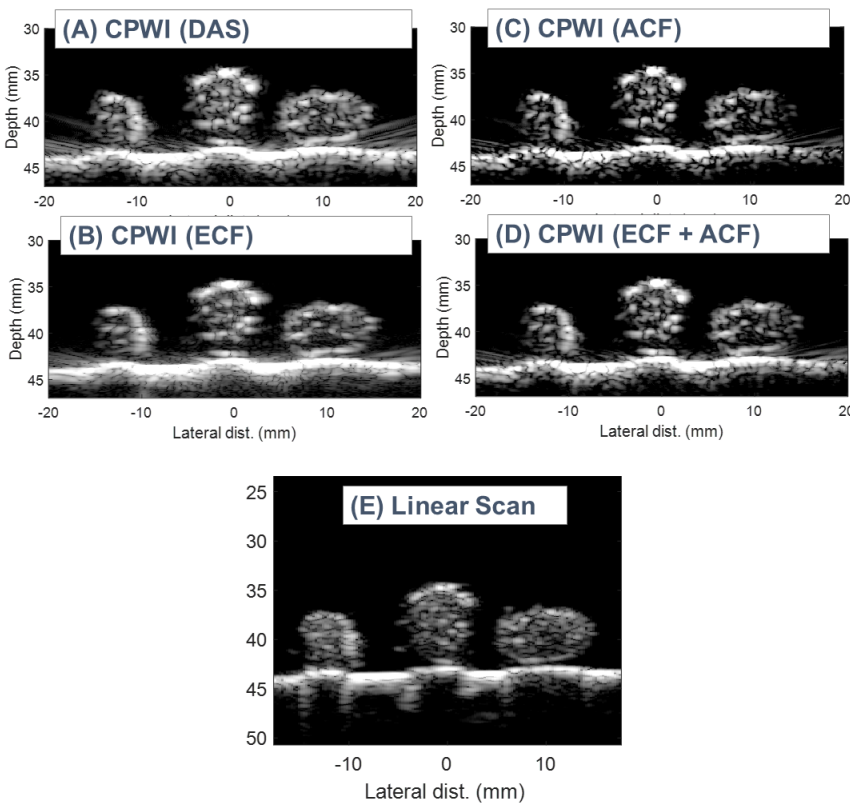

Fig. 3. (A) Compounded plane wave images (CPWI) with delay and sum (DAS) beamforming. (B) CPWI using element coherence factor (ECF). (C) CPWI using angular coherence factor (ACF) between steering angles. (D) CPWI using both ACF and ECF. (E) Conventional focused linear scan image. All figures are plotted with $40 \mathrm{~dB}$ dynamic range.

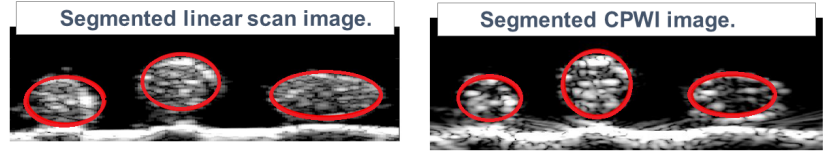

Fig. 4. Image shows the segmentation results. The red circles highlight the boundaries of the bone tissue identified by the segmentation algorithm. (Left) Segmented linear scan images. (Right) Segmented compounded plane wave images.

sum alone (Fig. 3 A) produced larger artefacts which are visible around the lateral extremes of the ultrasound images. The ECF method (Fig. 3 B) suppresses these artefacts at the lateral extremes, but the artefacts between bone samples still exist in the ultrasound images. The ACF method (Fig. 3 C) achieves a higher artefact suppression, however it may also incorrectly cancel the echoes from the bone samples. For example, in Fig. $3 \mathrm{C}$ the intensity of the bone sample (depth $=40 \mathrm{~mm}$, lateral $=15 \mathrm{~mm})$ is partially degraded . The ACF and ECF combination (Fig. 3 D) achieves the best artefact suppression without sacrificing the intensity of the bone samples. In Fig. $3 \mathrm{E}$, the linear scan method achieved the best SNR thanks to focusing with a peak SNR of $88 \mathrm{~dB}$, where the CPWI $(\mathrm{ACF}+\mathrm{ECF})$ had a peak SNR of $67 \mathrm{~dB}$.

\begin{tabular}{|l|c|c|}
\hline Estimation Error & Thickness estimation & Width estimation \\
\hline Linear scan & $26.3 \pm 10.1 \%$ & $12.3 \pm 8.8 \%$ \\
\hline CPWI (ACF+ECF) & $13.5 \pm 16.2 \%$ & $-6.3 \pm 12.5 \%$ \\
\hline
\end{tabular}

Fig. 5. Table shows the error rate for the bone dimension estimations. 
The segmentation results for the linear scan and CPWI (ACF + ECF), shown in Fig. 4, were compared with the ground truth to calculate the error rate for both methods. CPWI estimated the thickness and the width of the bone samples with a lower error rate than linear scan as listed in Fig. 5.

Overall, CPWI (ACF + ECF) had a better performance thanks to its wide steering range. The linear scan overestimated the size, which can be due to the widening of the regions outside the focal area. The most obvious difference between both methods is that the width is overestimated from the linear scan images and it was underestimated from the CPWI (ACF $+\mathrm{ECF})$ image.

\section{Discussion}

The beamforming algorithm based on delay and sum method may not be optimal for this study. To improve the image quality adaptive beamforming methods can be implemented. Nock et al. proposed a method to maximize the image intensity by re-aligning the phases of received signals [26]. Rodriguez-Molares et al. developed an adaptive ultrasound beamforming technique to image hard objects that takes into account the physics of specular reflection [27]. Minimum variance beamforming is another adaptive method that is widely employed in phased array and radar applications thanks to its high performance in the existence of uncertainties, such as imprecise knowledge of the arrival angle [28].

A limitation of most adaptive beamforming methods is the low performance in the presence of high speed of sound variations, such as between bone and soft-tissue. In this case, coherence-based methods can be used. This study already implemented two methods based on element-to-element coherence and angular coherence. In addition to these, coded excitation can be used to improve the SNR and image quality, which has been previously demonstrated for hard-tissue [29][31].

For the CPWI images, the dimension estimation had an error over $13 \%$ in thickness measurement, which is not negligible given that the experiments were performed under optimal conditions. In general, it is hard to characterize objects that are small compared to the imaging wavelength using ultrasound imaging. The point spread function (PSF) of the imaging system makes the objects appear larger than their actual size. Also, the reflections from surfaces orthogonal to the beam direction make it harder to find edges. To be able to estimate the dimensions accurately from ultrasound images, de-convolution, super-resolution or other filtering methods can be implemented [32]-[34].

Due to the coronavirus pandemic, it was not possible to repeat, refine and extend the experimental work in this study. However, given the encouraging ex vivo results, we want to optimize our imaging technique and start a human study to analyze the in vivo performance of plane wave imaging for detection and characterization of the fabella.

\section{CONCLUSiON}

The fabella is a sesamoid bone of both clinical and evolutionary significance. Although detectable by ultrasound, no studies have utilized this technique to gather information about this bone. Here, we use two methods of ultrasound imaging to investigate the ability of ultrasound to detect fabella presence/absence and quantify its dimensions. The compounded plane wave imaging (CPWI) beamformed using the combination of element coherence factor and angular coherence factor achieved the best performance thanks to its wide steering range. The linear scan overestimated the size, which can be due to the widening of the regions outside the focal area.

\section{REFERENCES}

[1] M. A. Berthaume and A. M. J. Bull, "Human biological variation in sesamoid bone prevalence: the curious case of the fabella," Journal of Anatomy, vol. 236, no. 2, pp. 228-242, 2020.

[2] M. A. Berthaume, E. Di Federico, and A. M. J. Bull, "Fabella prevalence rate increases over 150 years, and rates of other sesamoid bones remain constant: a systematic review," Journal of Anatomy, vol. 235, no. 1, pp. $67-79,2019$.

[3] P. Phukubye and O. Oyedele, "The incidence and structure of the fabella in a south african cadaver sample," Clin Anat, vol. 24, pp. 84-90, 2011.

[4] Y. Tabira, T. Saga, N. Takahashi, K. Watanabe, M. Nakamura, and K.I. Yamaki, "Influence of a fabella in the gastrocnemius muscle on the common fibular nerve in japanese subjects," Clin Anat, vol. 26, pp. 893902, 2012.

[5] A. Driessen, M. Balke, C. Offerhaus, W. J. White, S. Shafizadeh, C. Becher, B. Bouillon, , and J. Hoher, "The fabella syndrome - a rare cause of posterolateral knee pain: a review of the literature and two case reports," BMC Musculoskelet Disord, vol. 15, p. 100, 2014.

[6] T. Kim, H. Chung, H. Lee, Y. Choi, and J.-H. Son, "A case report and literature review on fabella syndrome after high tibial osteotomy," Medicine, vol. 97, p. e9585, 2018.

[7] W. Hou, L. Xu, J. Wang, B. Wang, L. Liu, K. Xu, Y. Cai, H. Guo, and $\mathrm{P}$. $\mathrm{Xu}$, "Fabellar prevalence, degeneration and association with knee osteoarthritis in the chinese population," Scientific Reports, vol. 9, p. 13046, 2019.

[8] Z. W. Jin, S. Shibata, H. Abe, Y. Jin, X. W. Li, and G. Murakami, "A new insight into the fabella at knee: the foetal development and evolution," Folia Morphol, vol. 76, pp. 87-93, 2017.

[9] S. Ehara, "Potentially symptomatic fabella: Mr imaging review," Jpn J Radiol, vol. 32, pp. 1-5, 2014.

[10] S.-X. Zeng, X.-L. Dong, R.-S. Dang, G.-S. Wu, J.-F. Wang, D. Wang, H.-L. Huang, and X.-D. Guo, "Anatomic study of fabella and its surrounding structures in a chinese population," Surg Radiol Anat, vol. 34, pp. 65-71, 2012.

[11] J. K. Sekiya, J. A. Jacobson, and E. M. Wojtys, "Sonographic imaging of the posterolateral structures of the knee: Findings in human cadavers," Arthroscopy, vol. 18, pp. 872-881, 2002.

[12] A. Cesmebasi, R. J. Spinner, J. Smith, S. M. Bannar, and J. T. Finnoff, "Role of sonography in the diagnosis and treatment of common peroneal neuropathy secondary to fabellae," J Ultrasound Med, vol. 35, pp. 441447, 2016.

[13] C. Corvalan, C. Tang, and M. Robinson, "Fabella and cyamella of the human knee joint: discovery by dissection and ultrasound examination," Eur J Anat, vol. 22, pp. 103-109, 2018.

[14] K. M. Dale, S. B. Boggess, B. Boggess, and C. T. Moorman, "Ultrasound evaluation and surgical excision of a fabella causing peroneal neuropathy in a track athlete," Case Reports in Orthopedics, vol. 2018, p. 2371947 , 2018.

[15] Q. Vallet, N. Bochud, C. Chappard, P. Laugier, and J.-G. Minonzio, "In vivo characterization of cortical bone using guided waves measured by axial transmission," IEEE Trans. Ultrason., Ferroelectr., Freq. Control, vol. 63, no. 9, pp. 1361-1372, 2016.

[16] C. Liu, B. Li, Q. Diwu, Y. Li, R. Zhang, D. Ta, and W. Wang, "Relationships of ultrasonic backscatter with bone densities and microstructure in bovine cancellous bone," IEEE Trans. Ultrason., Ferroelectr., Freq. Control, vol. 65, no. 12, pp. 2311-2321, 2018.

[17] Q. Grimal and P. Laugier, "Quantitative ultrasound assessment of cortical bone properties beyond bone mineral density," IRBM, vol. 40, pp. 16-24, 2019. 
[18] J. Schneider, D. Ramiandrisoa, G. Armbrecht, D. Felsenberg, K. Raum and J.-G. Minonzio, "In vivo measurements of cortical thickness and porosity at the proximal third of the tibia using guided waves: Comparison with site-matched peripheral quantitative computed tomography and distal high-resolution peripheral quantitative computed tomography," Ultrasound Med Biol, vol. 45, no. 5, pp. 1234-1242, 2019.

[19] K. A. Wear, "Mechanisms of interaction of ultrasound with cancellous bone: A review," IEEE Trans. Ultrason., Ferroelectr, Freq. Control, vol. 67, no. 3, pp. 454-482, 2020.

[20] H. N. Minh, J. Du, and K. Raum, "Estimation of thickness and speed of sound in cortical bone using multifocus pulse-echo ultrasound," IEEE Trans. Ultrason., Ferroelectr., Freq. Control, vol. 67, no. 3, pp. 568-579, 2020.

[21] E. Boni, L. Bassi, A. Dallai, F. Guidi, V. Meacci, A. Ramalli, S. Ricci, and P. Tortoli, "Ula-op 256: A 256-channel open scanner for development and real-time implementation of new ultrasound methods," IEEE Trans. Ultrason., Ferroelectr., Freq. Control, vol. 63, no. 10, pp. 1488 1495, 2016.

[22] E. Boni, L. Bassi, A. Dallai, V. Meacci, A. Ramalli, M. Scaringella, F. Guidi, S. Ricci, and P. Tortoli, "Architecture of an ultrasound system for continuous real-time high frame rate imaging," IEEE Trans. Ultrason., Ferroelectr., Freq. Control, vol. 64, no. 9, pp. 1276-1284, 2017.

[23] A. Stanziola, C. H. Leow, E. Bazigou, P. D. Weinberg, and M. Tang, "Asap: Super-contrast vasculature imaging using coherence analysis and high frame-rate contrast enhanced ultrasound," IEEE Transactions on Medical Imaging, vol. 37, no. 8, pp. 1847-1856, 2018.

[24] B. Zhuang, R. Rohling, and P. Abolmaesumi, "Accumulated angle factorbased beamforming to improve the visualization of spinal structures in ultrasound images," IEEE Trans. Ultrason., Ferroelectr., Freq. Control, vol. 65 , no. 2 , pp. 210-222, 2018.
[25] Z.-K. Huang and K.-W. Chau, "A new image thresholding method based on gaussian mixture model," Appl Math Comput, vol. 205, no. 2, pp. 899-907, 2008.

[26] L. Nock, G. E. Trahey, and S. W. Smith, "Phase aberration correction in medical ultrasound using speckle brightness as a quality factor," JASA, vol. 85, pp. 1819-1833, 1989.

[27] A. Rodriguez-Molares, A. Fatemi, L. Lovstakken, and H. Torp, "Specular beamforming," IEEE Trans. Ultrason., Ferroelectr., Freq. Control, vol. 64, no. 9, pp. 1285-1297, 2017.

[28] R. G. Lorenz and S. P. Boyd, "Robust minimum variance beamforming," IEEE Trans Signal Proces, vol. 53, pp. 1684-1696, 2005.

[29] S. Harput, T. Evans, N. Bubb, and S. Freear, "Diagnostic ultrasound tooth imaging using fractional Fourier transform," IEEE Trans. Ultrason., Ferroelectr., Freq. Control, vol. 58, no. 10, pp. 2096-2106, 2011.

[30] S. Harput, B. Raiton, J. R. McLaughlan, S. D. Evans, and S. Freear, "The periodicity between the aggregated microbubbles by secondary radiation force," in IEEE International Ultrasonics Symposium (IUS), 2011.

[31] S. Harput, J. McLaughlan, D. M. Cowell, and S. Freear, "New performance metrics for ultrasound pulse compression systems," in IEEE International Ultrasonics Symposium (IUS), 2014, pp. 440-443.

[32] T. Taxt and J. Strand, "Two-dimensional noise-robust blind deconvolution of ultrasound images," IEEE Trans. Ultrason., Ferroelectr., Freq. Control, vol. 48, no. 4, pp. 861-866, 2001.

[33] S. Harput, K. Christensen-Jeffries, A. Ramalli, J. Brown, J. Zhu, G. Zhang, C. H. Leow, M. Toulemonde, E. Boni, P. Tortoli, R. J. Eckersley, C. Dunsby, and M.-X. Tang, "3-d super-resolution ultrasound imaging with a 2-d sparse array," IEEE Trans. Ultrason., Ferroelectr., Freq. Control, vol. 67, no. 2, pp. 269-277, 2020.

[34] A. Bar-Zion, O. Solomon, C. Tremblay-Darveau, D. Adam, and Y. C. Eldar, "Sushi: Sparsity-based ultrasound super-resolution hemodynamic imaging," IEEE Trans. Ultrason., Ferroelectr., Freq. Control, vol. 65, no. 12 , pp. 2365-2380, 2018. 